\title{
A Model Based Approach for Estimating Aggregate Interference in Wireless Networks
}

\author{
Janne Riihijärvi and Petri Mähönen \\ Institute for Networked Systems, RWTH Aachen University \\ Kackerstrasse 9, D-52072 Aachen, Germany \\ email: \{jar,pma\}@inets.rwth-aachen.de
}

\begin{abstract}
In this paper we discuss the problem of estimating aggregate interference in large scale wireless networks. We argue that the usual approximations assuming uniform distribution of transmitters can lead into significant approximation errors. We then show how these approximation errors can be reduced by adopting a model based approach using spatial statistics of the actual transmitter distribution as part of the model. The resulting approach is still very lightweight numerically, and enables trading off computational complexity to approximation accuracy in a flexible manner. The developed methodology is general, and can be used for aggregate interference estimation both within individual wireless networks, and also between two or more wireless communication systems.
\end{abstract}

\section{INTRODUCTION}

Aggregate interference plays a key role in a number of resource management and optimization problems in wireless networks. There has been especially strong interest towards development of improved models of aggregate interference in the dynamic spectrum access (DSA) research community (see, for example, [1]-[5]). This is because most of the early work on DSA focused on interference management between primary and secondary systems assuming single active interference source, or concentrated on aggregate allowable interference from the primary system perspective only, which is obviously not sufficient for most applications. This leads to the question how multiple active secondary users distributed over larger spatial areas interfere with the primary system, which has been the focus of the aforementioned research.

Most of the existing work on aggregate interference estimation has assumed that the transmitters are uniformly distributed either over whole space, or outside some exclusion region in which transmissions are not allowed. For transmitters sufficiently far away from the point at which interference is to be estimated this is usually a very good approximation, provided that the overall density of interferers is properly chosen. However, the assumption of uniform distribution can lead to significant approximation errors when the distance to the point of interest is similar to typical distances between transmitters.

The origin of this approximation error can be easily understood using a cellular network like deployment as an example. At a point near the base station, due to the planned structure of the network, likelihood of being close to another base station is very small or non-existent. Nevertheless, in the uniform approximation there is a significant probability of one or more additional transmitters being nearby. This results in much higher variability of interference in the model than would occur in an actual deployment. For user deployed networks, such as femtocells, the approximation error usually goes another way around. Transmitters tend to be deployed in large clusters (such as apartment buildings), so being near to one transmitter implies that one is almost certainly near to other transmitters as well [6]. Again, the use of uniform approximation can easily lead into a significant approximation error.

In this paper we propose a model based approach for aggregate interference estimation. We use techniques from spatial statistics [7], [8] to construct a probabilistic model of the transmitter location distribution. From this model, we then approximate the contribution of the nearby transmitters to the aggregate interference distribution, and add to this the contribution from the far away transmitters using the uniform assumption. Our approach is a combination of analytical and numerical techniques, since for non-uniform transmitter distributions analytically tractable solutions are typically available only in asymptotic regimes not of interest here. We also discuss the conditions indicating when this is formally allowed.

The rest of this paper is structured as follows. In Section II we give a concise overview of those aspects of spatial statistics needed for our approximation of aggregate interference. Then in Section III we show through a small case study the improvement our approach brings to the usual uniform transmitter distribution approach. Finally, we draw the conclusions and outline future work in Section IV.

\section{Spatial Statistics of Point Processes}

The mathematical framework for modeling and reasoning about transmitter locations is that of point processes [9], [10]. A point process can be thought of as a random variable, each realization of which yields a pattern of transmitters in a given region. We will give in the following a concise summary of the basic definitions underlying point process theory, to the extent needed for our particular application.

We consider some compact region $W \subset \mathbb{R}^{2}$ over which our transmitter distributions will be defined. Formally a point process $N$ is now a random counting measure on $W$. This means that for each sufficiently regular (technically, measurable) subregion $A \subset W, N(A)$ is an integer-valued random 

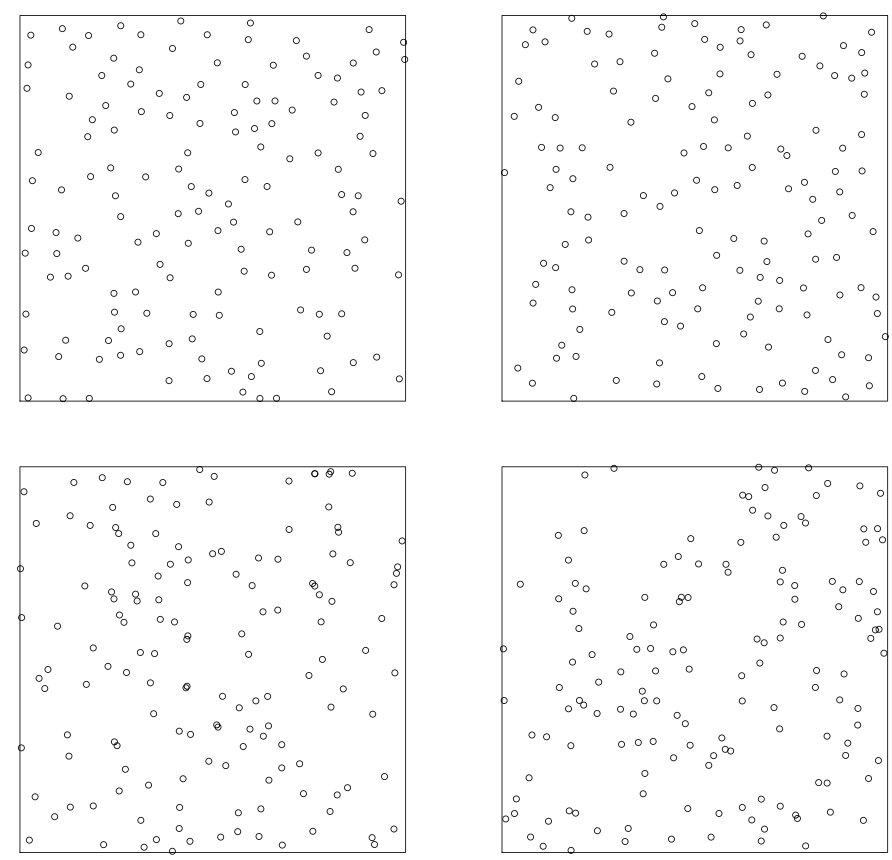

Fig. 1. Realizations of the Geyer saturation process with $\gamma=0, \gamma=0.25$ (top panels), $\gamma=0.75$ and $\gamma=1$ (bottom panels).

variable counting the number of points or node locations within $A$. The joint probability distributions of $N\left(A_{k}\right)$ for different regions $A_{k} \subseteq W$ serve to define the point process completely. The simplest example is given by the uniform assumption mentioned in the introduction. Technically, a point process $N_{\lambda}$ is said to be a homogeneous Poisson point process of intensity $\lambda$, if $N_{\lambda}(A)$ is Poisson with parameter $\lambda|A|$, where $|A|$ is the area of $A$, and $N_{\lambda}(A)$ and $N_{\lambda}(B)$ are independent for any disjoint $A$ and $B$. This results in locations that are uniformly distributed in $W$, and are independent of each other.

We can specify more complex point process models by defining a density function with respect to the distribution of a Poisson point process, usually further chosen to have $\lambda=1$. Such a density is defined on the space of counting measures on $W$, and in essence quantifies how much more or less likely a given pattern of node locations is to occur in the model compared to the Poisson case. We have shown in [8] that the Geyer saturation process [11], a generalisation of the Strauss process [10], yields very good fits for a wide variety of data sets on transmitter locations. For the Strauss process we have the density

$$
f(N)=\alpha \beta^{\#(N)} \gamma^{s_{r}(N)},
$$

where $s_{r}(N)$ is the number of point pairs of $N$ that are closer than distance $r$ apart. For the Geyer process an additional saturation threshold $\zeta$ is added, bounding the contribution of the exponent of $\gamma$. The case $\zeta \rightarrow \infty$ yields the Strauss process as a limit. Especially the parameter $\gamma$ has an intuitive interpretation. If $\gamma=0$, the likelihood of a point pattern arising with any pair of points closer than distance $r$ apart becomes zero. Therefore, the process becomes an example of a pure hard-core process. As $\gamma$ is increased, close by point pairs become possible, but are still less likely to occur than for the Poisson case. Finally, $\gamma=1$ yields the Poisson point process as a limit. Increasing $\gamma$ further will then yield clustered distributions, as discussed for the user deployed networks case in the introduction. Figure 1 shows example realizations of the Geyer process for different values of the parameter $\gamma$. The increased regularity in the structure of the point process as $\gamma$ is reduced is clearly visible.

Various statistics can be defined on point processes, succinctly characterizing different aspects of them. For our purposes here, the most important ones are nearest neighbor distances. For a point process $N$, the nearest neighbor distance distribution function $G_{N}(r)$ is defined as the cumulative distribution function of the distance from a randomly selected point of $N$ to its nearest neighbor. In the Poisson point process case this can be explicitly computed as

$$
G(r)=1-\exp \left(-\lambda \pi r^{2}\right)
$$

For the general case of Gibbs process with density $f$, additional definitions are needed. We define the Papangelou conditional intensity [12] by

$$
\lambda^{*}(x, N) \equiv \frac{f(N \cup x)}{f(N)}
$$

for points $x \notin N$, and by

$$
\lambda^{*}(x, N) \equiv \frac{f(N)}{f(N-x)}
$$

otherwise. Then the intensity of $N$, giving the average number of points over unit area, becomes $\lambda_{N} \equiv \mathbb{E}\left\{\lambda^{*}(x, N)\right\}$, and the nearest neighbor distance distribution function is given by

$$
G(r)=\frac{1}{\lambda} \mathbb{E}\left\{\lambda^{*}(0, N) 1[N(B(0, r)) \neq 0]\right\},
$$

where $1[\cdot]$ is the indicator function [12]. We denote the $k$ th order analogs of $G_{N}(r)$ by $G_{N}^{[k]}(r)$, with $G_{N}^{[1]}(r) \equiv G_{N}(r)$. These are the cumulative distribution functions for $k$ th nearest neighbor distances. Finally, we define the second order product density of $N$ by

$$
\rho_{N}(x, y) \equiv \mathbb{E}\left\{\lambda^{*}(x, N) \lambda(y, N \cup\{x\})\right\} .
$$

This essentially measures the likelihood of there being a node at $y$ given that there is a node at $x$.

Higher order nearest neighbor distance distributions functions are known explicitly for a number of simple point process models, see, for example, [13] and [14] for discussion and references.

\section{AgGRegate InTERFERENCE FROM ORdER STATISTICS OF NeIGHBOR Distances}

We begin by discussing the influence of the transmitter distribution on the dominant components of aggregate interference. Figure 2 shows the distribution of interference power from nearest neighboring transmitter, measured at the proximity of a randomly selected transmitter, for both the uniformly random (Poisson) case, as well as for the Geyer model 

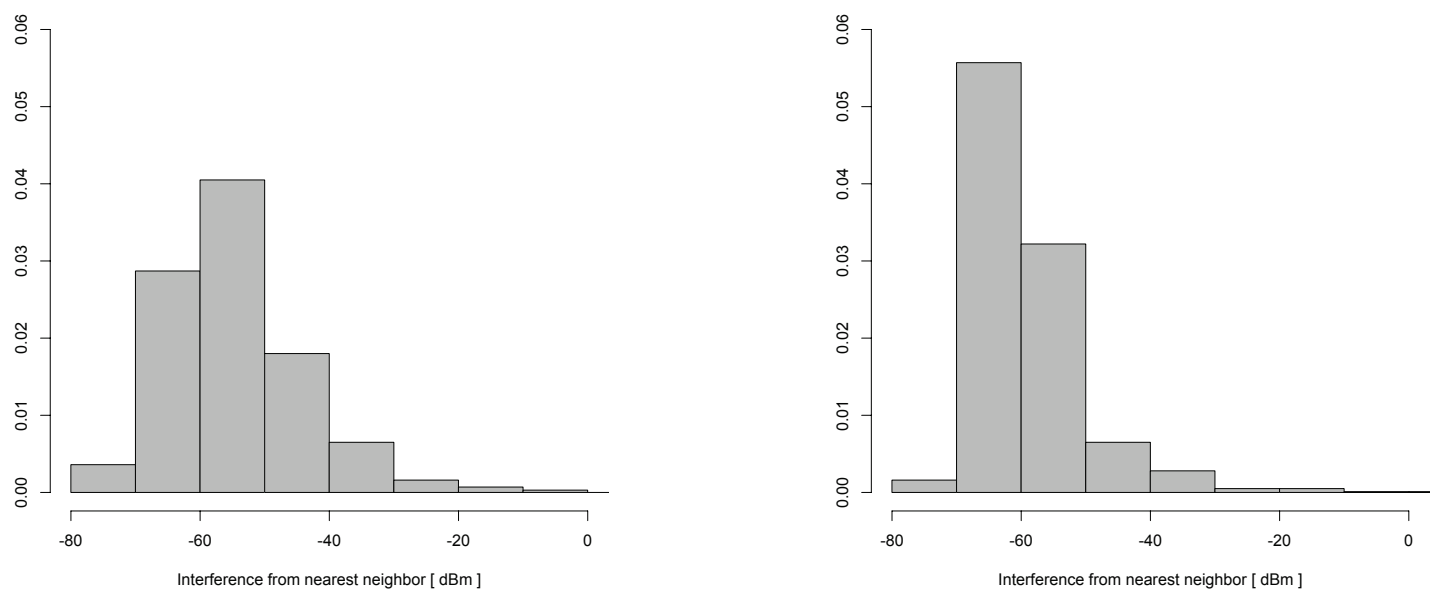

Fig. 2. Distribution of nearest-neighbor interference for Poisson (left) and the fitted Geyer (right) models of same density.
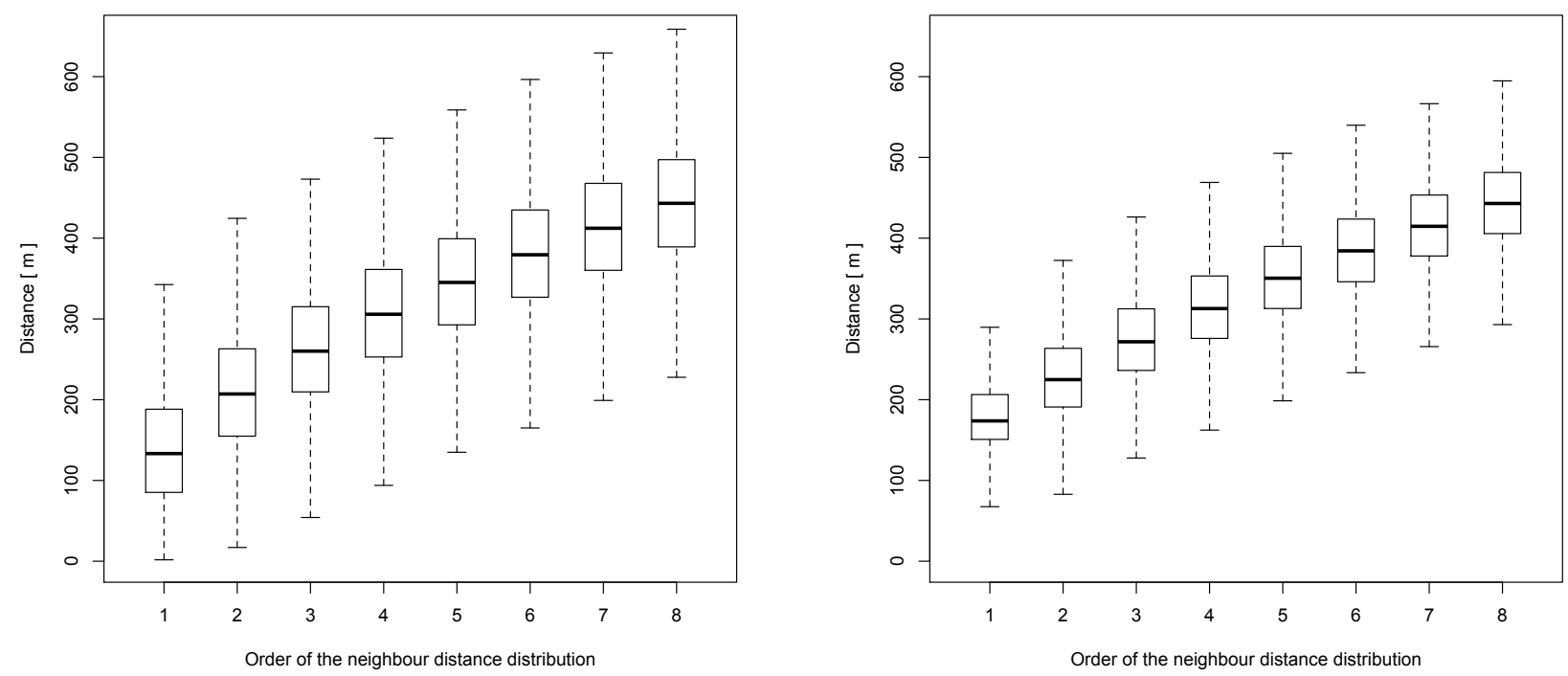

Fig. 3. Distributions $G^{[k]}(r)$ of the $k$ th nearest-neighbor distances for Poisson (left) and the fitted Geyer (right) models of the same density. The three horizontal lines of the box correspond to the median and the $25 \%$ and $75 \%$ quantiles of the data, with the whiskers giving the data point at most 1.5 times the interquartile range from the edge of the box.

fitted to the Wi-Fi network structure given in [8]. The impact of the regularity captured well by the Geyer model is very clear. The adoption of the Poisson model as an approximation would lead into both overestimating the interference caused, and overestimating the variability of the nearest neighbor interference. The figure was obtained assuming transmit power of $1 \mathrm{~W}$, using the Xia-Bertoni propagation model [15] defined for a given frequency $f$ (expressed in $\mathrm{GHz}$ ) and distance $d$ (in kilometers) by

$$
L(d)=K+A_{1} \log _{10}(f)+A_{2} \log _{10}(d),
$$

where the constants $K, A_{1}$ and $A_{2}$ are taken to have values of $131.1 \mathrm{~dB}, 21 \mathrm{~dB}$ and $37.6 \mathrm{~dB}$, respectively (see [16] for detailed discussion on the determination of these coefficients for the chosen propagation model). Operating frequency of $2 \mathrm{GHz}$ was also assumed. We shall use this simple propagation and transmitter configuration model throughout in the following. Additional randomness due to shadowing environment and/or fast fading can be incorporated to the propagation model without difficulty.

The simplest way to quantify the differences between the two models shown in Figure 2, and to extend the discussion 
into larger neighborhoods from the nearest neighbor case, is to study the order statistics $G_{N}^{[k]}(r)$ for the two models. Figure 3 shows the estimates of these, obtained from extensive Monte Carlo simulations (for the Geyer model no analytical solution exists to the best of our knowledge, making numerical estimates necessary). Note that for higher $k$ the behavior of the inter-point distances is quite similar to the nearest neighbor case discussed above. The Poisson approximation results in higher variation and underestimation of the mean for small $k$, but approximation error does become smaller and smaller as $k$ becomes large. The latter property is formally a consequence of the second order product density of the Geyer model tending to that of a Poisson point process as $x$ and $y$ are taken to be wide apart.

The convergence of $k$ th nearest neighbour distances suggests a simple approximation scheme for the aggregate interference. Instead of simulating large network instances from the node location models, which can become computationally intensive, we can sample from the (marginal) distance distributions directly. Therefore, the expected aggregate interference is approximated as a series

$$
\mathbb{E}\{I\} \approx \sum_{k=1}^{N(W)} \int P(r) d G_{N}^{[k]}(r)
$$

where $P(r)$ is the received power at distance $r$ computed using the selected path loss model and transmit power, and the integral is understood in the Stieltjes sense. Higher order statistics can be approximated similarly. Interference at other locations can be likewise estimated by appropriate change of the distance distribution functions $G^{[k]}$ in (8). For example, at random locations independent on the structure of $N$, the order statistics of the empty space function $F(r)$ can be used [10].

This approximation ignores the potentially complicated joint component of the distributions, but would be expected to result in moderately accurate approximation of the leading order contributions. Further, due to the convergence property of the second order product density, it should be sufficient to use the more accurate distributions arising from the Geyer model only for small values of $k$, and use the analytically soluble Poisson inter-point distance distributions or approximate aggregate interference results directly for higher orders. Formally, we can therefore write our approximation as

$$
\mathbb{E}\{I\} \approx \sum_{k=1}^{O} \int P(r) d G_{N}^{[k]}(r)+\sum_{k=O+1}^{N(W)} \int P(r) d G_{\text {Poisson }}^{[k]}(r),
$$

where $O$ denotes the order of the approximation. As discussed above, the latter sum can also be replaced with any of the approximate aggregate interference results from the literature. Figure 4 shows selected results from these estimates, indicating that using only the 2-3 lowest order distributions from the Geyer case suffices for good approximations. Notice that the figure shows results for interference from a relatively small neighborhood of eight nodes only, since the given approximation converges already in that case quite rapidly.

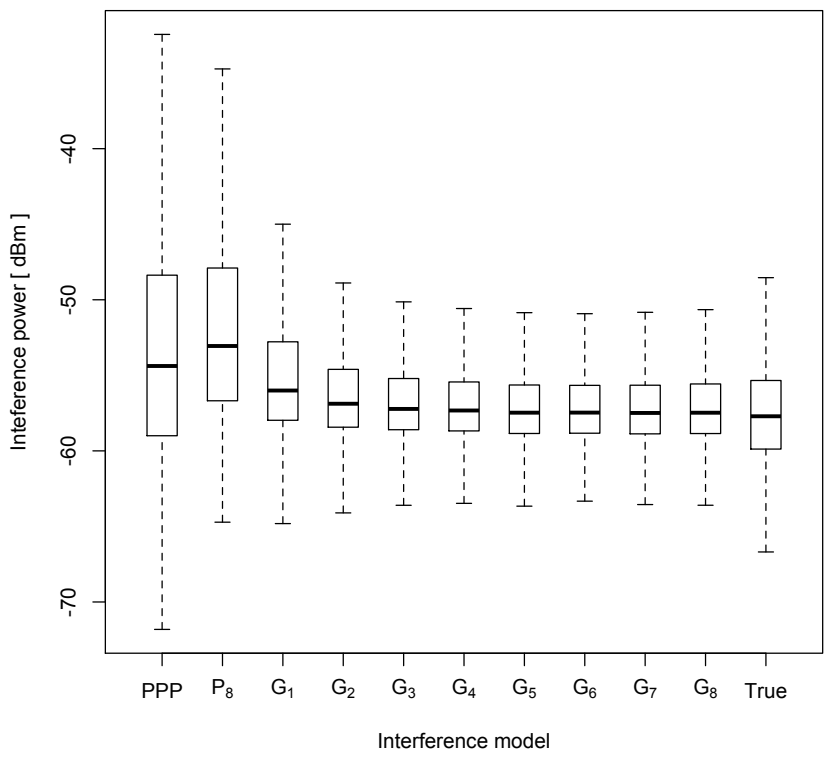

Fig. 4. Approximating the aggregate interference from eight nearest neighbors in networks with node locations following Geyer saturation model using order statistics for $k$ th nearest neighbour distances. The actual interference values are labeled "PPP" for the Poisson approximation, and "True" for the Geyer case, results for latter obtained using Monte Carlo simulations. " $P_{8}$ " denotes approximation for the Poisson case using order statistics of distance distributions only, and " $G_{k}$ " denotes the approximation introduced in the text, with order $k$. Conventions for the box plot are as in Figure 3.

For larger neighborhoods additional interference power from far away nodes is very well approximated by the Poisson case and the approximations in the literature can again be used directly.

This process can be made even more light-weight by approximating the low- $k$ order distance distributions by suitably fitted probability distribution functions. Figure 5 illustrates the results from using the logistic distribution with cumulative distribution function

$$
F(x)=\frac{1}{1+\exp (-(x-\mu) / s)}
$$

for $k \in\{1,2,3\}$ for this purpose, with parameter values obtained by maximum likelihood fitting to the empirical distance distributions. Despite the simplicity of this approach, we see from the figure that the maximum approximation error is for most cases on the order of few $\mathrm{dB}$, and that the Poisson approximation is rather poor except for the very lowermost tail of the interference distribution. These results can undoubtedly be improved further by more careful choice of the distribution function used in the approximation.

\section{CONCLUSIONS}

In this paper we discussed the problem of estimating aggregate interference in wireless networks, making two major contributions. First, we have shown through numerical examples 


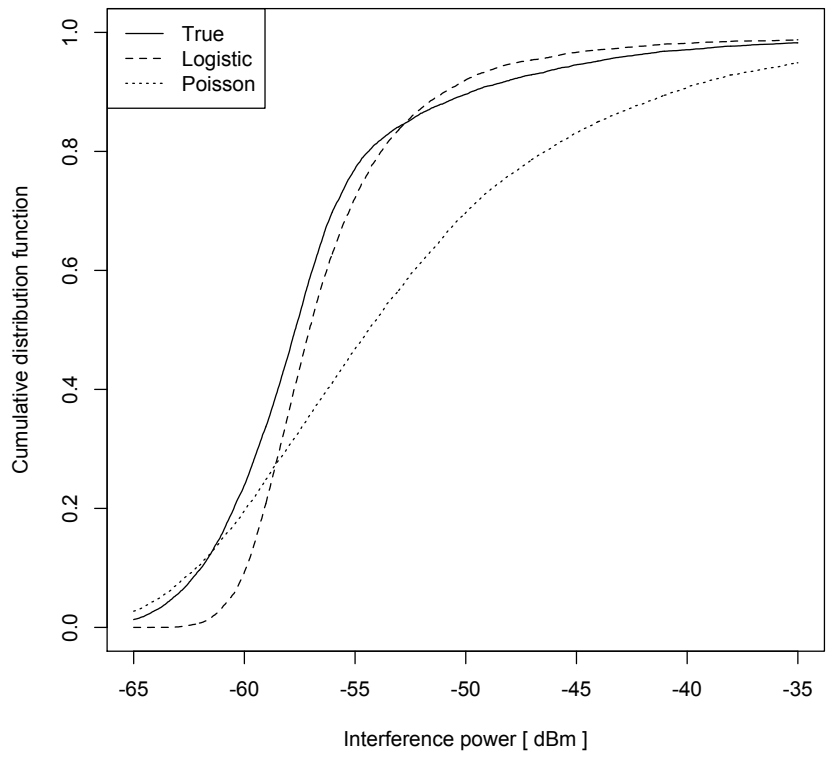

Fig. 5. Cumulative distribution function of aggregate interference in Geyer networks, approximation using logistic distribution for inter-node distances, and results from approximating the whole network structure by a homogeneous Poisson point process.

that the commonly used approach of approximating transmitter locations with a uniform distribution or a homogeneous Poisson point process can result in significant approximation errors. Therefore, great care should be applied whenever modeling an arbitrary transmitter distribution with a Poisson process, especially since large-scale wireless networks are known to show significant departures from the uniform structure [6], [8]. Second, we proposed a model-based approach for estimating the distribution of aggregate interference, in which a Gibbs point process model is fitted to the transmitter distribution, and the order statistics of the nearest neighbor distances of the models are used to estimate the contribution of the nearby transmitters to aggregate interference. Contributions from far away nodes can still be computed using the prevalent Poisson assumption without significant loss of accuracy due to the properties of the used Gibbs models. This results in a scheme that is lightweight to implement numerically, and can result in significant improvements in the accuracy of the estimated interference distribution. We have found that the results are also relatively insensitive to small changes in the parameters of the Gibbs process used. This indicates that for practical applications the relevant distance distribution functions can be estimated in advance for a small family of models, and the selection between the models can be then done on-line.

\section{ACKNOWLEDGEMENT}

The work reported in this contribution was carried out with partial financial support from the European Commission under the QUASAR project. We also thank RWTH Aachen University and the German Research Foundation (Deutsche Forschungsgemeinschaft, DFG) for providing financial support through the UMIC research centre.

\section{REFERENCES}

[1] A. Ghasemi and E. Sousa, "Interference aggregation in spectrum-sensing cognitive wireless networks," Selected Topics in Signal Processing, IEEE Journal of, vol. 2, no. 1, pp. 41-56, 2008.

[2] M. Aljuaid and H. Yanikomeroglu, "A cumulant-based characterization of the aggregate interference power in wireless networks," in Vehicular Technology Conference (VTC 2010-Spring), 2010 IEEE 71st. IEEE, 2010, pp. 1-5.

[3] X. Hong, C. Wang, and J. Thompson, "Interference modeling of cognitive radio networks," in Vehicular Technology Conference, 2008. VTC Spring 2008. IEEE. IEEE, 2008, pp. 1851-1855.

[4] K. Sung, M. Tercero, and J. Zander, "Aggregate interference in secondary access with interference protection," IEEE Communications letters, vol. 15, no. 6, pp. 629-631, 2011.

[5] A. Rabbachin, T. Quek, H. Shin, and M. Win, "Cognitive network interference," Selected Areas in Communications, IEEE Journal on, vol. 29, no. 2, pp. 480-493, 2011.

[6] J. Riihijärvi, P. Mähönen, and M. Rübsamen, "Characterizing wireless networks by spatial correlations," IEEE Communications Letters, vol. 11, no. 1, pp. 37-39, January 2007.

[7] N. Cressie, Statistics for spatial data. Wiley-Interscience, 1993.

[8] J. Riihijärvi and P. Mähönen, "Modeling Spatial Structure of Wireless Communication Networks," in INFOCOM IEEE Conference on Computer Communications Workshops, 2010, 2010, pp. 1-6.

[9] A. F. Karr, Point Processes and Their Statistical Inference, 2nd ed. Marcel Dekker, 1991.

[10] D. Stoyan, W. S. Kendall, and J. Mecke, Stochastic geometry and its applications. Wiley, 1995.

[11] C. Geyer, "Likelihood inference for spatial point processes," Stochastic Geometry: Likelihood and Computation, vol. 80, pp. 79-140, 1999.

[12] J. Møller and R. Waagepetersen, Statistical inference and simulation for spatial point processes. CRC Press, 2004, vol. 100.

[13] M. Haenggi, "On distances in uniformly random networks," Information Theory, IEEE Transactions on, vol. 51, no. 10, pp. 3584-3586, 2005.

[14] S. Srinivasa and M. Haenggi, "Distance distributions in finite uniformly random networks: Theory and applications," Vehicular Technology, IEEE Transactions on, vol. 59, no. 2, pp. 940-949, 2010.

[15] L. Maciel, H. Bertoni, and H. Xia, "Unified approach to prediction of propagation over buildings for all ranges of base station antenna height," IEEE transactions on vehicular technology, vol. 42, no. 1, pp. 41-45, 1993.

[16] H. Xia, "A simplified analytical model for predicting path loss in urban and suburban environments," IEEE Transactions on Vehicular Technology, vol. 46, no. 4, pp. 1040-1046, November 1997. 